\title{
LA ORIENTACIÓN VOCACIONAL EN LOS COLEGIOS PÚBLICOS Y PRIVADOS DE LIMA: SITUACIÓN ACTUAL Y PROPUESTA DE UN PROGRAMA DE ACCIÓN PARA LA SECUNDARIA PÚBLICA
}

\author{
Augusto EmiLIO FrISANCHO LeÓN*
}

\begin{abstract}
RESUMEN
El objetivo principal del presente estudio fue determinar si existían o no diferencias en la forma de llevar a cabo la orientación vocacional de sus estudiantes entre los colegios públicos y privados de Lima Metropolitana. Aplicando un cuestionario a una muestra de 279 alumnos -entre hombres y mujeres- que acababan de terminar su educación secundaria y que se encontraban realizando estudios preuniversitarios, se llegó a establecer que sí hay diferencias: la orientación vocacional que reciben los alumnos en los colegios públicos es más pobre (contempla menos aspectos) y se lleva a cabo de manera casi puntual (sólo en 5to. o, a lo más, en 4to. y 5to. de secundaria) que la recibida por los alumnos de los colegios privados. Además, los alumnos de los colegios públicos califican mayormente de «regular» la orientación vocacional recibida, mientras que los alumnos de los colegios privados la califican principalmente de «buena».
\end{abstract}

Adicionalmente a estos resultados, el presente estudio plantea un programa de orientación vocacional para ser realizado con los estudiantes de los colegios públicos, programa que busca ser integral y llevarse a cabo a lo largo de los tres últimos años de la secundaria.

Palabras clave: Orientación vocacional; Colegios públicos; Colegios privados; Lima; Programa de orientación vocacional.

\section{ABSTRACT}

This plan's main objective is to determinate if the developing of vocational guide for students is going to be the same or different between private and public high schools from Lima Metropolitana.

After a question paper application to a sample of 279 -male and female- just ending high school students, we found several differences between the two kinds of high schools.

In addition of that finding, this plan proposes a Vocational guide plan direct to public high students, for the last three degrees in particular.

Keywords: Vocational guide, Public high schools, Private high schools, Lima, Vocational guide plan.

* Docente Auxiliar, Facultad de Psicología de la UNMSM. E-mail: aufril56@yahoo.es 


\section{INTRODUCCIÓN}

La orientación vocacional se considera una parte integral del proceso educativo del individuo que se realiza con la finalidad de facilitarle una ayuda que le conduzca a encontrar la máxima concordancia posible entre sus capacidades, actitudes, valores e intereses y las aptitudes necesarias para las diversas opciones que presenta el mundo laboral, de tal manera que se encamine hacia la autorrealización personal.

Si bien la orientación vocacional se realiza en casi todos los colegios privados y en la mayoría de los colegios públicos, hay un desconocimiento de lo que realmente se lleva a cabo en dichos colegios, particularmente en los públicos. Al parecer, en los centros educativos del Estado la orientación vocacional es experimentada por los alumnos más como un hecho puntual, aislado, separado de lo educativo y sin ligazón con un proyecto personal de vida, que lo que realmente debe ser: un proceso, el cual debe llevarse a cabo durante toda la educación secundaria y con una base en la educación primaria.

En el Diseño Curricular Básico de Educación Secundaria se menciona que los dos principales objetivos de este nivel educativo apuntan a la formación integral del estudiante y a su ubicación en el mundo laboral. Sin embargo, la educación que realmente recibe el alumno en la escuela no ofrece ni lo uno ni lo otro. Su educación es parcializada y está más enfocada a lo meramente instructivo que a lo formativo. Y si bien desde hace cuatro años se instituyó una hora de tutoría grupal en todos los años de la educación secundaria, esta tutoría es conducida por profesores no capacitados en orientación educativa, que no cuentan con un plan de trabajo y que no realizan en la práctica una labor de tutoría.

Todo lo anterior hace que los estudiantes al terminar su educación secundaria no cuenten con proyecto personal de vida y, por lo tanto, no sepan qué hacer en adelante. Esta indefinición les va a ocasionar pérdidas de tiempo e innecesarias frustraciones.

Por lo anterior, el presente trabajo se propuso describir la manera cómo se está llevando a cabo la orientación vocacional en los colegios de la secundaria pública, compararla con la forma como ésta es realizada en los colegios privados y, finalmente, proponer un plan de acción para que la orientación vocacional pueda llevarse adecuadamente en los colegios públicos.

El problema de investigación al que trató de dar respuesta la presente investigación fue el siguiente:

¿Existen diferencias en la forma de llevar a cabo la orientación vocacional de sus estudiantes entre los colegios públicos y privados de Lima Metropolitana?

\section{Tipo de investigación}

Investigación de tipo descriptivo comparativa. 


\section{Marco teórico}

Existen algunos modelos que describen el proceso de orientación vocacional. Uno es el planteado por Lázaro y Asensi (1987). Él plantea tres fases o etapas: el diagnóstico, la información profesional y el consejo. Otro modelo es el planteado por Álvarez y otros (1994), quienes proponen tres etapas: la obtención de información (sobre sí mismo, sobre el sistema educativo y sobre el mundo profesional), el análisis y reflexión y la toma de decisión.

Para la presente investigación se propone un modelo de Orientación Vocacional basado en el modelo de Álvarez y otros (1994), pero con modificaciones. Este modelo se esquematiza de la siguiente manera:

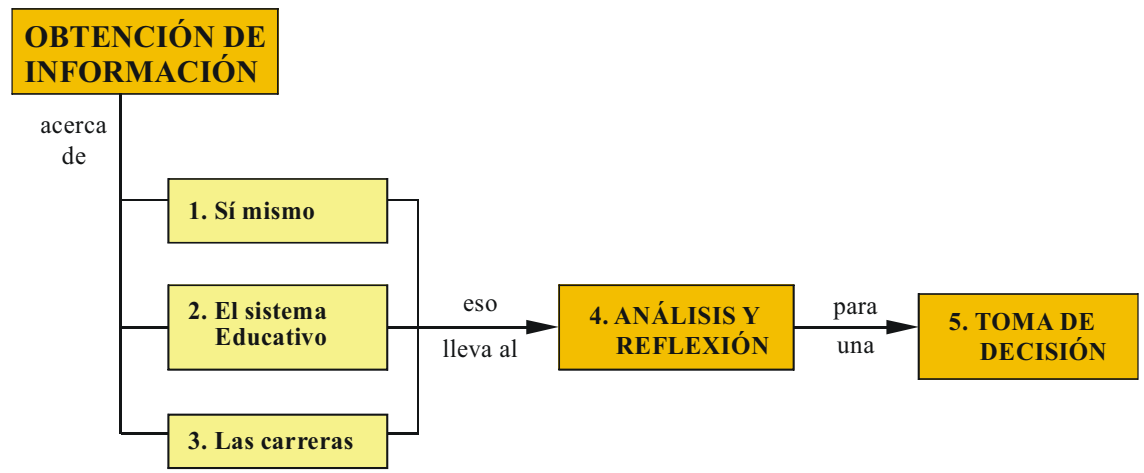

\section{Hipótesis}

Existen diferencias en la forma de llevar a cabo la orientación vocacional de sus estudiantes entre los colegios públicos y privados de Lima Metropolitana.

\section{MÉTODOS}

\section{Población y muestra}

La población estuvo constituida por los alumnos matriculados en el Ciclo Extraordinario 2003-2004 del Centro Preuniversitario de San Marcos. Fueron aproximadamente 2000 los alumnos inscritos en dicho ciclo. El ciclo extraordinario es solamente para aquellos alumnos que acaban de terminar el 5to. Año de secundaria. Se aseguró, de esta manera, que la información sobre la orientación vocacional recibida sea reciente.

La muestra inicial fue de 307 alumnos. Luego de depurar algunos cuestionarios mal llenados, la muestra final quedó constituida por 279 estudiantes, de los cuales 94 provenían de colegios públicos y 185 de colegios privados.

\section{Tipo de muestreo}

Se realizó un muestreo intencional. Se escogieron para contestar al cuestionario a los alumnos de seis aulas elegidas al azar. 


\section{Técnica de recolección de datos}

Cuestionario elaborado para el propósito de la investigación.

Técnica de análisis de datos

Estadísticos descriptivos: frecuencias y porcentajes.

\section{RESULTADOS}

Los resultados a los que arribó la presente investigación se presentan a continuación. Presentaré las respuestas dadas a las preguntas principales del cuestionario y posteriormente haré un análisis e interpretación de las mismas.

Cuadro 1. Pregunta 3: ¿Recibiste en tu colegio orientación vocacional?

\begin{tabular}{|l|c|c|c|c|}
\cline { 2 - 5 } \multicolumn{1}{c|}{} & \multicolumn{2}{c|}{ Sí } & \multicolumn{2}{c|}{ No } \\
\hline \multicolumn{1}{c|}{ Colegios } & f & \% & f & $\%$ \\
\hline Públicos & 74 & $\mathbf{7 8 . 7}$ & 20 & $\mathbf{2 1 . 3}$ \\
\hline Privados & 164 & $\mathbf{8 8 . 6}$ & 21 & $\mathbf{1 1 . 4}$ \\
\hline
\end{tabular}

Públicos $(n=94)$

Privados $(n=185)$

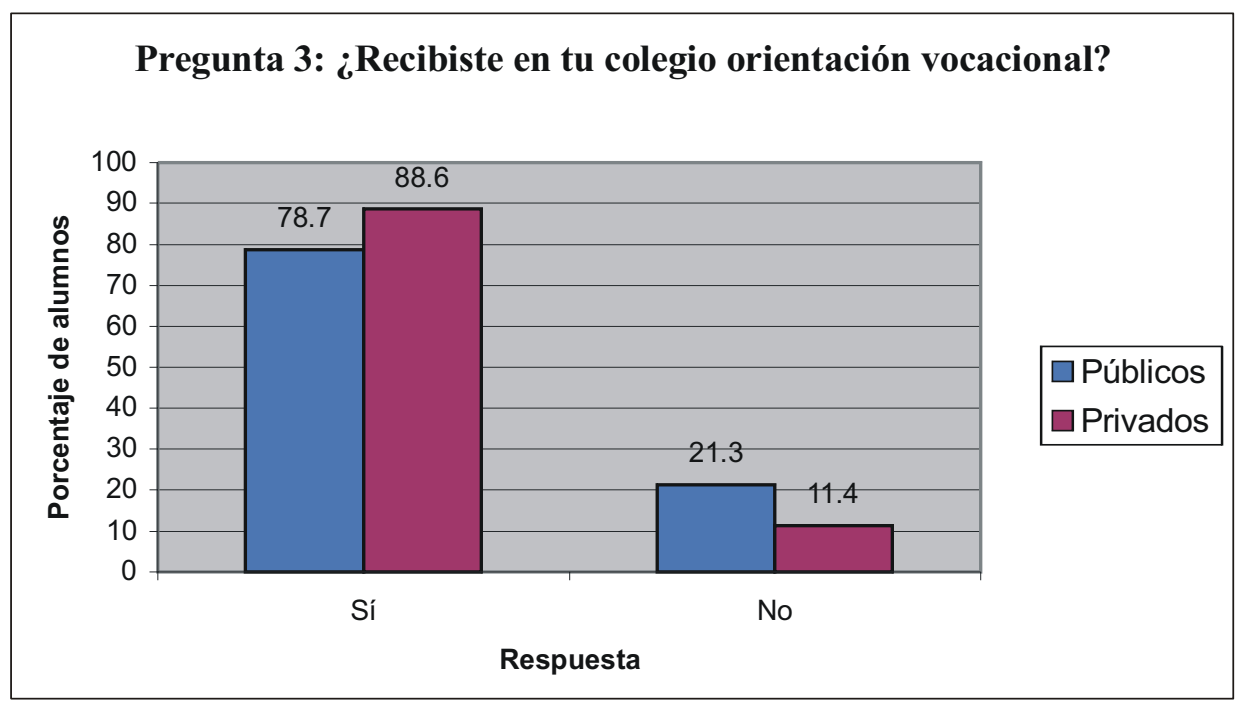


En el Cuadro 1 y en el gráfico se aprecia que el porcentaje de alumnos de los colegios, tanto públicos como privados, que afirma haber recibido orientación vocacional en su colegio, es alto $(78.7 \%$ y $88.6 \%$, respectivamente). Sin embargo, hay un porcentaje preocupante de alumnos de colegio públicos $(21.3 \%)$ que afirma no haber recibido orientación vocacional en la institución educativa donde estudió.

Cuadro 2. Pregunta 5: ¿En qué momento recibiste la orientación vocacional?*

\begin{tabular}{|c|c|c|c|c|c|c|c|c|c|c|}
\cline { 2 - 11 } \multicolumn{1}{c|}{} & \multicolumn{3}{c|}{$5^{\circ}$ año } & \multicolumn{2}{c|}{$4^{\circ}$ año } & \multicolumn{2}{c|}{$3^{\circ}$ año } & \multicolumn{2}{c|}{$4^{\circ}-\mathbf{5}^{\circ}$ años } & \multicolumn{2}{c|}{$3^{\circ}-\mathbf{4}^{\circ}-\mathbf{5}^{\circ}$ años } \\
\hline Colegios & F & $\%$ & f & $\%$ & f & \% & f & $\%$ & F & $\%$ \\
\hline Públicos & 30 & $\mathbf{4 0 . 5}$ & 4 & $\mathbf{5 . 4}$ & 1 & $\mathbf{1 . 4}$ & 31 & $\mathbf{4 1 . 9}$ & 8 & $\mathbf{1 0 . 8}$ \\
\hline Privados & 49 & $\mathbf{2 9 . 9}$ & 2 & $\mathbf{1 . 2}$ & 0 & $\mathbf{0 . 0}$ & 77 & $\mathbf{4 7 . 0}$ & 32 & $\mathbf{1 9 . 5}$ \\
\hline
\end{tabular}

* Entre los alumnos de los colegios privados hubo 4 (2.4\%) que marcaron una alternativa diferente a las mencionadas.

Públicos $(n=74)$

Privados $(n=164)$

\section{Pregunta 5: ¿En qué momento recibiste la orientación vocacional?}

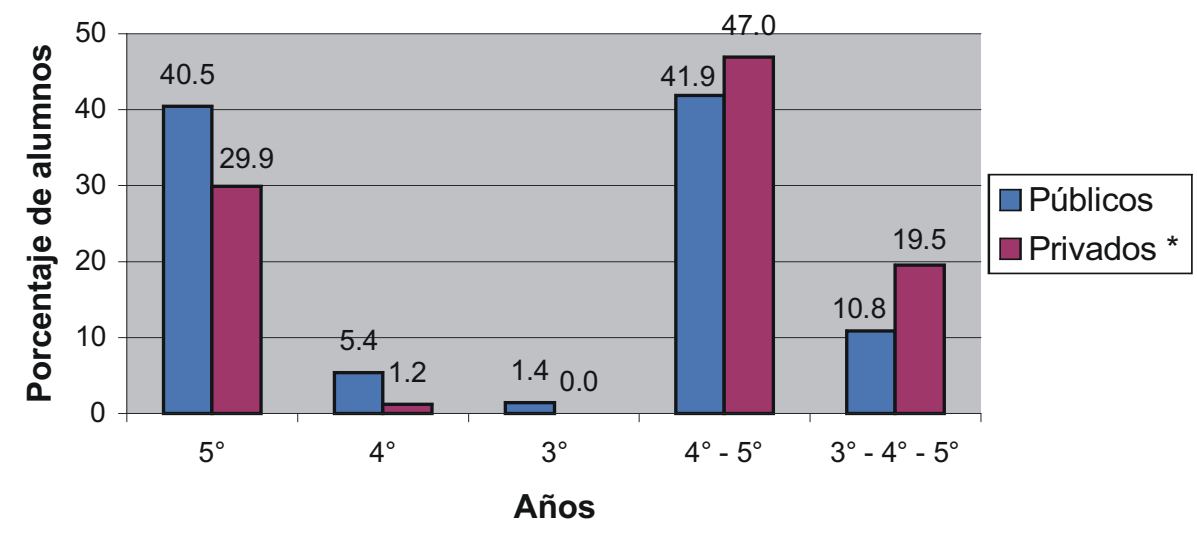

En el Cuadro 2 y en el gráfico se puede observar que tanto en los colegios públicos como en los privados los alumnos están recibiendo orientación vocacional en 4to. y en 5to. año de secundaria (41.9\% y $47.0 \%$, respectivamente). Se aprecia que la orientación vocacional está comenzando a ser vista más como un proceso que como un hecho puntual. Sin embargo, todavía hay un porcentaje alto, sobre todo en los colegios públicos $(40.5 \%)$ que realizan la orientación vocacional sólo en 5to. año de secundaria. Lo ideal es que la orientación vocacional se empezara a dar en 3ro. de secundaria y terminara en 5to. Pero esto sólo se da en un $19.5 \%$ de los colegios privados y en un escaso $10.8 \%$ de los colegios públicos. 
Cuadro 3. Pregunta 6: La orientación vocacional que recibiste consistió en:

\begin{tabular}{|l|c|c|c|c|c|c|c|c|c|c|}
\cline { 2 - 10 } \multicolumn{1}{c|}{} & \multicolumn{2}{c|}{$\begin{array}{c}\text { Un } \\
\text { aspecto }\end{array}$} & \multicolumn{2}{c|}{$\begin{array}{c}\text { Dos } \\
\text { aspectos }\end{array}$} & \multicolumn{2}{c|}{$\begin{array}{c}\text { Tres } \\
\text { aspectos }\end{array}$} & \multicolumn{2}{c|}{$\begin{array}{c}\text { Cuatro } \\
\text { aspectos }\end{array}$} & \multicolumn{2}{c|}{$\begin{array}{c}\text { Cinco } \\
\text { aspectos }\end{array}$} \\
\hline Colegios & f & $\mathbf{\%}$ & f & $\mathbf{\%}$ & f & \% & f & \% & f & \% \\
\hline Públicos & 26 & $\mathbf{3 5 . 1}$ & 18 & $\mathbf{2 4 . 3}$ & 18 & $\mathbf{2 4 . 3}$ & 11 & $\mathbf{1 4 . 9}$ & 1 & $\mathbf{1 . 4}$ \\
\hline Privados & 18 & $\mathbf{1 1 . 0}$ & 39 & $\mathbf{2 3 . 8}$ & 54 & $\mathbf{3 2 . 9}$ & 34 & $\mathbf{2 0 . 7}$ & 19 & $\mathbf{1 1 . 6}$ \\
\hline
\end{tabular}

Públicos $(n=74)$

Privados $(n=164)$

Sí mismo: Información sobre aspectos del sujeto (nivel intelectual, personalidad, intereses, etc.). Sistema educativo: Información sobre las alternativas de estudio que ofrece el sistema educativo peruano.

Profesiones: Información sobre las carreras, en qué consisten y qué condiciones y capacidades se deben tener para estudiarlas.

Análisis y reflexión: Realización de actividades de análisis y reflexión sobre la información obtenida.

Toma de decisión: Entrenamiento en toma de decisiones para la elección de carrera.

\section{Pregunta 6: La orientación vocacional que recibiste consistió en:}

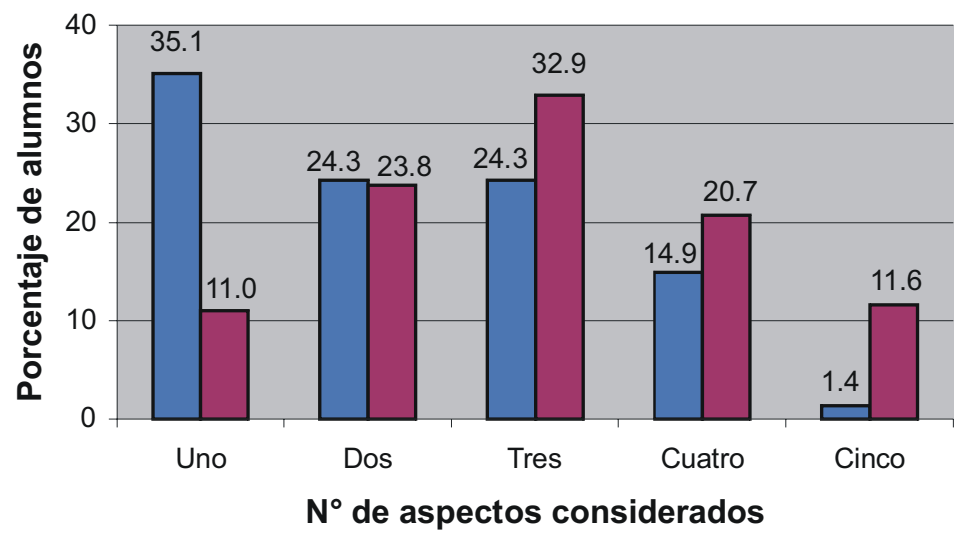

Para el análisis del Cuadro 3 y del gráfico correspondiente se parte del supuesto que una orientación vocacional será mejor cuanto más aspectos comprenda. Según el modelo asumido, son cinco los aspectos que debe reunir una orientación vocacional: información sobre sí mismo, información sobre el sistema educativo, información sobre las profesiones, análisis y reflexión, y toma de decisión.

Se aprecia que un $35.1 \%$ de los alumnos de colegios públicos ha recibido un solo aspecto de la orientación vocacional. Si esto se compara con el $32.9 \%$ de los alumnos de colegios 
privados que manifiesta haber recibido tres aspectos de la orientación vocacional, la diferencia en cuanto a la calidad de la orientación vocacional proporcionada por los colegios salta a la vista. Lo ideal es que la orientación vocacional ofrecida por los colegios contemple los cinco aspectos señalados en el párrafo anterior, pero como se puede apreciar, sólo el 1.4\% de los alumnos de colegios públicos dice haber realizado esos cinco aspectos, contra el ya significativo $11.6 \%$ de los colegios privados.

Cuadro 4. Pregunta 7: ¿Cómo calificarías la orientación vocacional recibida en tu colegio?

\begin{tabular}{|l|c|c|c|c|c|c|c|c|c|c|}
\cline { 2 - 10 } \multicolumn{1}{c|}{} & \multicolumn{2}{c|}{ Deficiente } & \multicolumn{2}{c|}{ Mala } & \multicolumn{2}{c|}{ Regular } & \multicolumn{2}{c|}{ Buena } & \multicolumn{2}{c|}{ Excelente } \\
\hline Colegios & f & $\mathbf{\%}$ & $\mathbf{f}$ & $\mathbf{\%}$ & $\mathbf{f}$ & $\mathbf{\%}$ & $\mathbf{f}$ & $\mathbf{\%}$ & $\mathbf{f}$ & $\mathbf{\%}$ \\
\hline Públicos & 1 & $\mathbf{1 . 4}$ & 6 & $\mathbf{8 . 1}$ & 37 & $\mathbf{5 0 . 0}$ & 28 & $\mathbf{3 7 . 8}$ & 2 & $\mathbf{2 . 7}$ \\
\hline Privados & 1 & $\mathbf{0 . 6}$ & 3 & $\mathbf{1 . 8}$ & 52 & $\mathbf{3 1 . 7}$ & 90 & $\mathbf{5 4 . 9}$ & 18 & $\mathbf{1 1 . 0}$ \\
\hline
\end{tabular}

Públicos $(n=74)$

Privados $(n=164)$

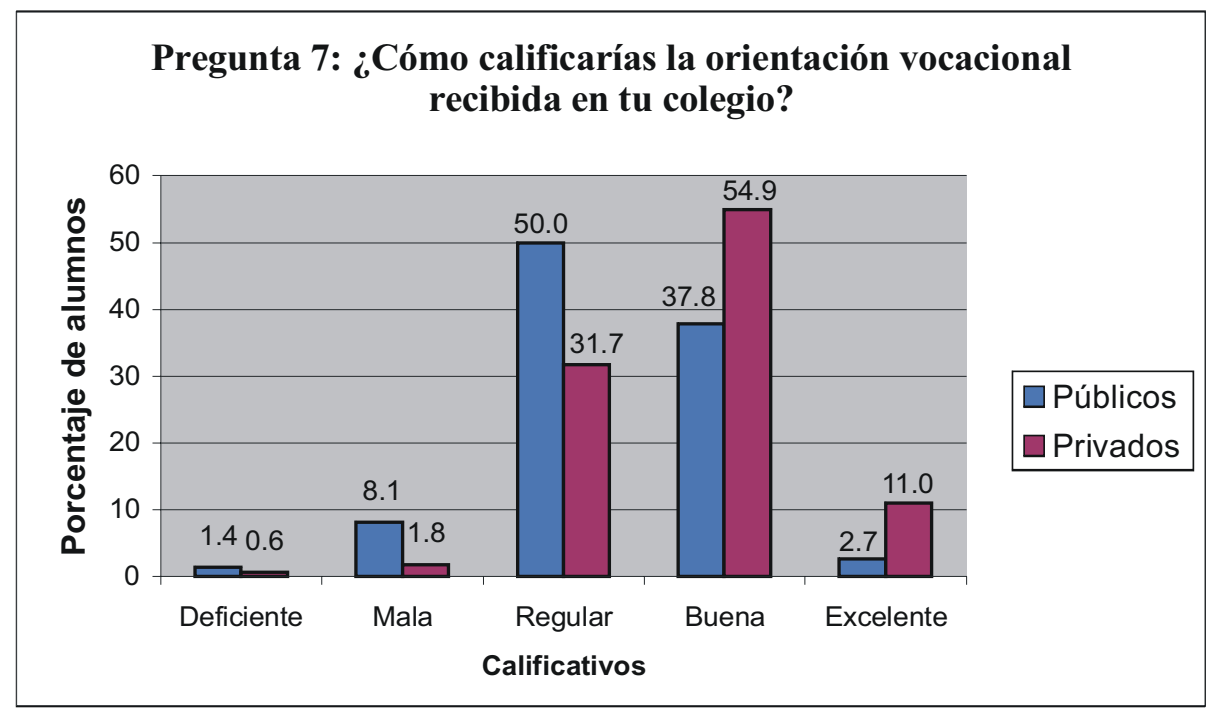

En el Cuadro 4 y en el gráfico se puede apreciar que los alumnos de colegios públicos califican la orientación vocacional recibida como regular en un 50\% y como buena en un $37.8 \%$. En cambio, los alumnos de colegios privados la califican como buena en un $54.9 \%$ y como regular en un $31.7 \%$. Es claro que hay que mejorar la orientación vocacional que se ofrece en los colegios públicos de Lima Metropolitana. 
Cuadro 5. Pregunta 12: ¿Cuál es la postura de tus padres respecto a tu futuro vocacional?

\begin{tabular}{|l|c|c|c|c|c|c|c|c|}
\cline { 2 - 9 } \multicolumn{1}{c|}{} & \multicolumn{2}{c|}{ Impositivos } & \multicolumn{2}{c|}{ Colaborativos } & \multicolumn{2}{c|}{ Permisivos } & \multicolumn{2}{c|}{ Indiferentes } \\
\hline Colegios & f & $\mathbf{\%}$ & $\mathbf{F}$ & $\mathbf{\%}$ & $\mathbf{f}$ & $\mathbf{\%}$ & f & $\%$ \\
\hline Públicos & 6 & $\mathbf{6 . 4}$ & 76 & $\mathbf{8 0 . 8}$ & 12 & $\mathbf{1 2 . 8}$ & 0 & $\mathbf{0 . 0}$ \\
\hline Privados & 8 & $\mathbf{4 . 3}$ & 143 & $\mathbf{7 7 . 3}$ & 33 & $\mathbf{1 7 . 8}$ & 1 & $\mathbf{0 . 6}$ \\
\hline
\end{tabular}

Públicos $(n=94)$

Privados $(n=185)$

Impositivos: Me imponen sus criterios y preferencias.

Colaborativos: Colaboran y dialogan conmigo.

Permisivos: Dejan que lo decida yo solo.

Indiferentes: Se muestran indiferentes.

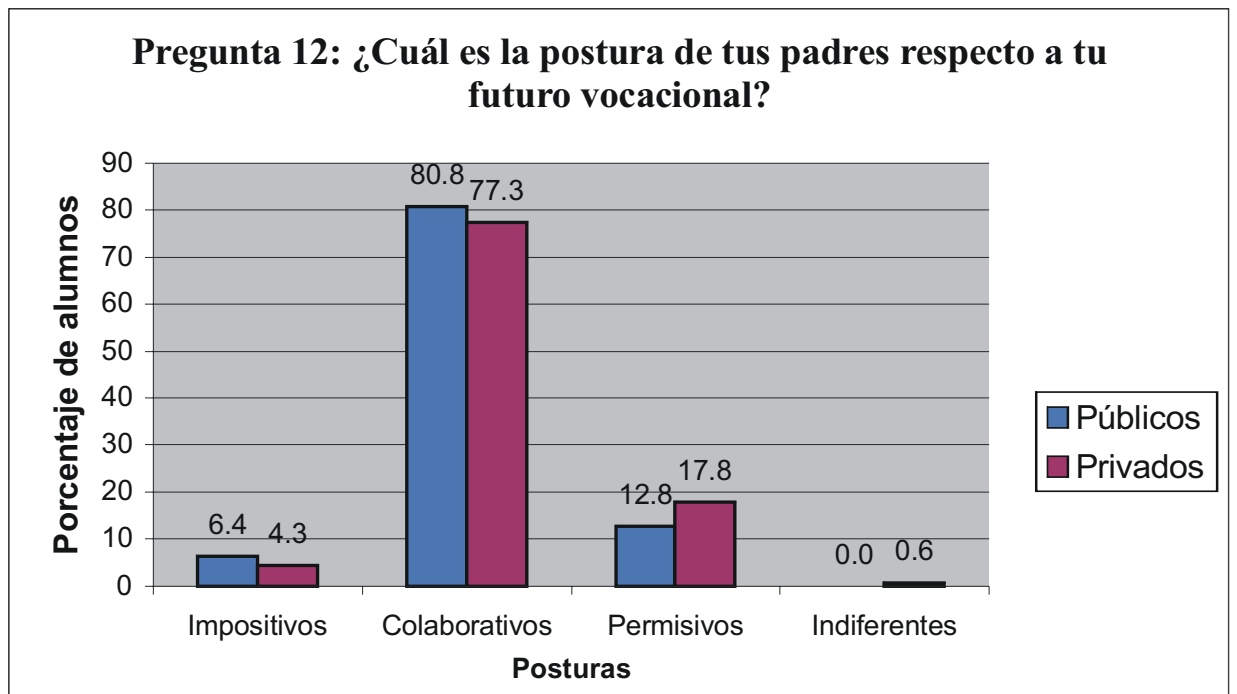

En el Cuadro 5 y en el gráfico se observa que tanto en los colegios públicos como privados la postura de los padres frente al futuro vocacional de sus hijos es mayormente de colaboración y diálogo $(80.8 \%$ y $77.3 \%$, respectivamente). 
Cuadro 6. Pregunta 14: ¿Qué importancia tienen para ti las siguientes razones al momento de elegir una carrera?

\begin{tabular}{|c|c|c|c|c|c|c|c|c|c|c|c|c|c|c|}
\hline \multirow[b]{2}{*}{ Colegios } & \multicolumn{2}{|c|}{ Vocación } & \multicolumn{2}{|c|}{ Dinero } & \multicolumn{2}{|c|}{ Capacidad } & \multicolumn{2}{|c|}{ Padres } & \multicolumn{2}{|c|}{ País } & \multicolumn{2}{|c|}{ Empleo } & \multicolumn{2}{|c|}{ Prestigio } \\
\hline & f & $\%$ & f & $\%$ & f & $\%$ & f & $\%$ & f & $\%$ & f & $\%$ & f & $\%$ \\
\hline Públicos & 59 & 62.7 & 6 & 6.4 & 23 & 24.5 & 1 & 1.1 & 2 & 2.1 & 2 & 2.1 & 1 & 1.1 \\
\hline Privados & 97 & 52.4 & 17 & 9.2 & 58 & 31.4 & 1 & 0.6 & 3 & 1.6 & 6 & 3.2 & 3 & 1.6 \\
\hline
\end{tabular}

Públicos $(n=94)$

Privados $(n=185)$
Vocación: Considero que es mi verdadera vocación.

Dinero: La carrera está bien remunerada (se gana bien).

Capacidades: La profesión es compatible con mis capacidades e intereses.

Padres: Cumpliría con el deseo de mis padres.

País: Es la profesión que el país necesita.

Empleo: Hay mayores posibilidades de conseguir empleo.

Prestigio: La profesión es bien vista socialmente (tiene prestigio).

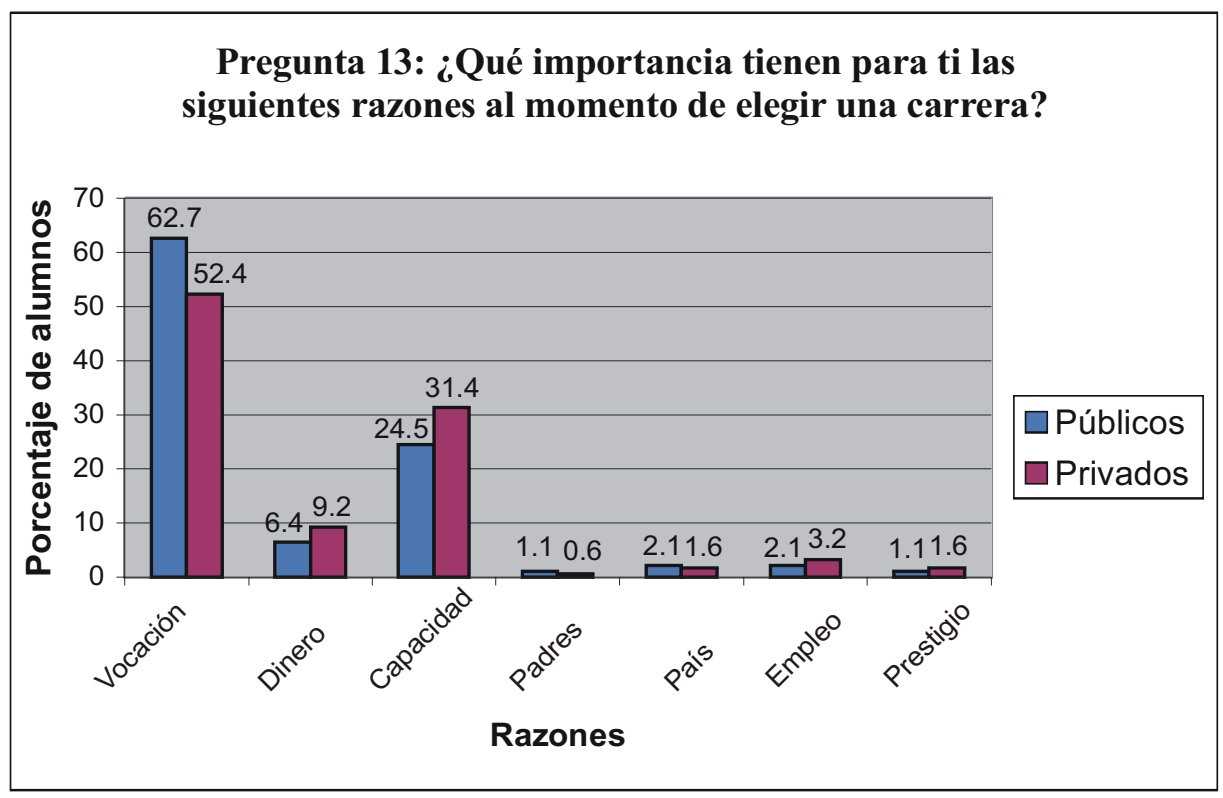

En el Cuadro 6 y en el gráfico respectivo se observa que la razón que la mayoría de los estudiantes de colegios públicos y privados considera más importante al momento de elegir una profesión u ocupación es «Considero que es mi verdadera vocación» $(62.7 \%$ y $52.4 \%$, respectivamente). En cambio, sólo el $24.5 \%$ de los alumnos de colegios públicos y el $31.4 \%$ de los privados, considera que "La profesión es compatible con mis capacidades e intereses», es el razón más importante al momento de elegir profesión u ocupación. Nosotros consideramos que esta última razón es la que debe primar al momento de elegir por una profesión. 


\section{CONCLUSIONES}

1. Se confirma la hipótesis de investigación de que existen diferencias en la forma de llevar a cabo la orientación vocacional de sus estudiantes entre los colegios públicos y privados de Lima Metropolitana.

2. La orientación vocacional en los colegios privados se realiza de manera más completa que en los colegios públicos. En la mayoría de los primeros, la orientación cubre tres de los cinco aspectos que debería contemplar, mientras que en la mayoría de los colegios públicos se cubre escasamente un solo aspecto.

3. El ideal de que la orientación vocacional se vea como un proceso y que empiece a realizarse en 3er. año para terminar en 5to. año de secundaria se está dando más en los colegios privados que en los públicos. Por otra parte, si bien en ambos tipos de colegios la orientación vocacional se está impartiendo principalmente en dos años consecutivos (4to. y 5to. de secundaria), hay todavía un gran porcentaje de colegios públicos que realizan la orientación vocacional sólo en 5to. año de secundaria.

4. La mayoría de los estudiantes de los colegios públicos califica la orientación vocacional recibida de «regular» y un porcentaje menor la califica de «buena». En cambio, la mayoría de los estudiantes de los colegios privados califica la orientación vocacional recibida de «buena» y un porcentaje menor la califica de «regular».

\section{REFERENCIAS BIBLIOGRÁFICAS}

1. Álvarez, M. et al. (1994). La orientación vocacional a través del currículum y de la tutoría. Barcelona: ICE, Graó.

2. Bautista, R. et al. (1992). Orientación e intervención educativa en secundaria. Málaga: Aljibe.

3. Lázaro, A.; Asensi, J. (1987). Manual de orientación escolar y tutoría. Madrid: Narcea.

4. Martín, E.; Tirado, V. (2000). La orientación educativa y profesional en la educación secundaria. Barcelona: ICE, Horsori.

5. Rivas, F. (1995). Manual de asesoramiento y orientación vocacional. Madrid: Síntesis. 


\section{ANEXO \\ PROPUESTA DE PROGRAMA DE ORIENTACIÓN VOCACIONAL PARA LA SECUNDARIA PÚBLICA}

\section{Características de la propuesta}

- El programa planteado considera la orientación para la elección de carrera como un proceso y no como una actividad esporádica y aislada.

- El programa pone énfasis especial en otorgar al estudiante un papel activo en dicho proceso.

- El diseño, elaboración y evaluación del programa de orientación para la elección de carrera estará a cargo del equipo formado por el Coordinador de tutoría, los tutores y el psicólogo del centro (si lo hubiera).

- La ejecución y supervisión de dicho programa debe ser responsabilidad exclusiva del tutor.

- El programa considera también que es fundamental la participación activa del Director del centro educativo y de los padres de familia, desde el inicio hasta la culminación del mismo.

\section{Dinámica del proceso}

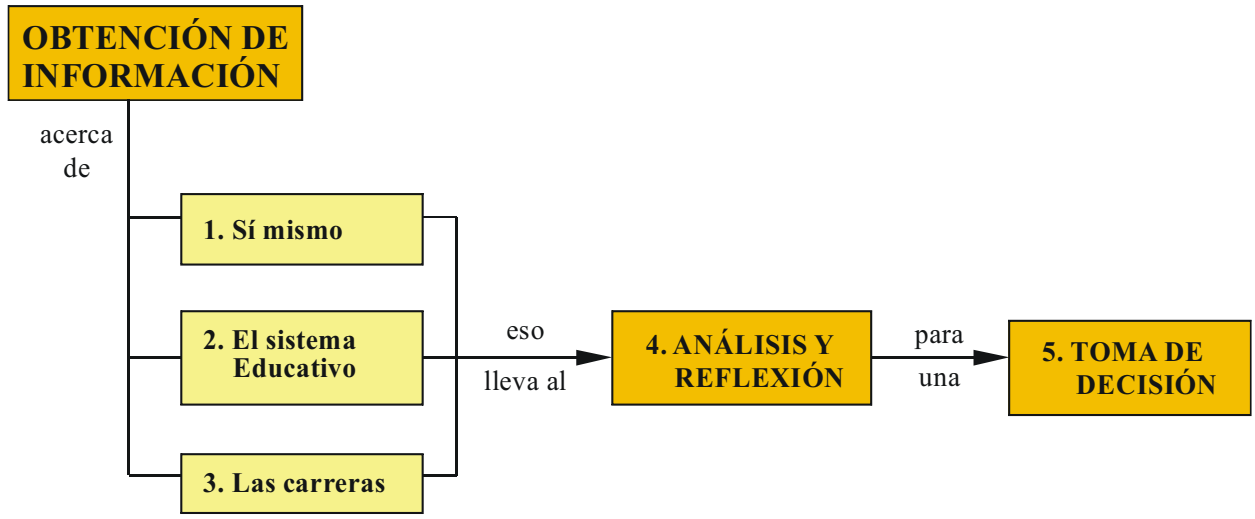

3. Estructura del programa

3.1. Objetivos.

3.2. Secuencia de acciones y actividades.

3.3. Métodos y técnicas de trabajo.

3.4. Materiales y equipos.

3.5. Evaluación. 


\subsection{Objetivos}

- Ayudar al alumno en el conocimiento de sí mismo (personalidad, intereses, aptitudes, etc.), de la estructura del sistema educativo y de las carreras.

- Conducir al alumno a una situación de análisis y reflexión sobre sí mismo, su experiencia educativa, su proyecto personal y la posibilidad de acceder a los estudios de una carrera.

- Capacitar al alumno para la toma de decisión en la elección de carrera como consecuencia del proceso anterior. Igualmente, en su propio sistema de valores, en el concepto de sí mismo y en el nivel de aspiración personal y familiar.

\subsection{Secuencia de acciones y actividades}

Hay una serie de acciones y actividades que se pueden programar en un programa de orientación vocacional. Algunas de ellas se mencionan a continuación:

- Aplicación de una encuesta o cuestionario vocacional.

- Aplicación de tests psicológicos de orientación vocacional.

- Informe verbal o escrito de los resultados de los tests.

- Información sobre las posibilidades de estudio y/o trabajo al término de la secundaria.

- Información sobre los Centros Educativos Ocupacionales (CEOs), Institutos Tecnológicos, Universidades, etc.

- Visita a los CEOs, Institutos Tecnológicos, Universidades, etc.

- Información sobre las profesiones u ocupaciones y sus requisitos para estudiarlas.

- Charlas a cargo de profesionales representativos de las distintas profesiones u ocupaciones.

- Charlas sobre el mercado laboral en el Perú (oferta y demanda de empleo).

- Generar reflexión, análisis y discusión sobre la información recibida acerca de sí mismos, los centros de formación y las profesiones u ocupaciones.

- Hacer que comparen la información recibida acerca de sí mismos con los requisitos de las profesiones u ocupaciones que son de su interés.

- Proporcionar orientación y consejo para el análisis de la información recibida.

- Proporcionar entrenamiento en toma de decisiones con la finalidad de que puedan elegir una profesión u ocupación.

- Hacer que relacionen sus alternativas de elección de carrera con su proyecto de vida.

- Hacer que elijan la profesión u ocupación que se adecúe más a sus capacidades y proyecto de vida.

Las actividades y acciones señaladas, además de otras más que los tutores y alumnos puedan proponer deben ser planificadas para ser llevadas a cabo durante 3ro., 4to. y 5to. de secundaria. Una secuencia de acciones y actividades podría ser la siguiente: 


\begin{tabular}{|c|c|c|c|c|c|}
\hline Año & $\begin{array}{c}\text { Recabar } \\
\text { información y } \\
\text { tomar } \\
\text { conocimiento }\end{array}$ & $\begin{array}{c}\text { Realización } \\
\text { de visitas }\end{array}$ & $\begin{array}{l}\text { Realización de } \\
\text { investigaciones }\end{array}$ & $\begin{array}{c}\text { Entrenamiento } \\
\text { en toma de } \\
\text { decisiones }\end{array}$ & $\begin{array}{c}\text { Tutoría } \\
\text { individual }\end{array}$ \\
\hline 3ro. & $\begin{array}{l}\text { Mediante charlas } \\
\text { sobre el programa } \\
\text { a realizar. }\end{array}$ & $\begin{array}{l}\text { A centros } \\
\text { laborales de } \\
\text { la localidad. }\end{array}$ & & & Sí \\
\hline 4to. & $\begin{array}{l}\text { A través de la } \\
\text { evaluación de su } \\
\text { personalidad, } \\
\text { aptitudes e } \\
\text { intereses. }\end{array}$ & $\begin{array}{l}\text { A centros de } \\
\text { instrucción } \\
\text { de la } \\
\text { localidad. }\end{array}$ & $\begin{array}{l}\text { Bibliográficas } \\
\text { y documentales } \\
\text { sobre las } \\
\text { carreras. }\end{array}$ & $\begin{array}{l}\text { En diferentes } \\
\text { situaciones } \\
\text { cotidianas. }\end{array}$ & Sí \\
\hline 5to. & $\begin{array}{l}\text { Mediante charlas } \\
\text { acerca del } \\
\text { sistema educativo } \\
\text { peruano. }\end{array}$ & & $\begin{array}{l}\text { De campo } \\
\text { sobre las } \\
\text { carreras. }\end{array}$ & $\begin{array}{l}\text { Para la elección } \\
\text { de la carrera. }\end{array}$ & Sí \\
\hline
\end{tabular}

\subsection{Métodos y técnicas de trabajo}

Dependiendo de la naturaleza de la acción o actividad a realizar, se podrá recurrir a:

- Exposiciones.

- Investigaciones o trabajos de campo.

- Debates.

- Análisis de casos.

\subsection{Materiales y equipos}

- Separatas.

- Dípticos o trípticos.

- Guías profesiográficas.

- Escalas de observación.

- Tests psicológicos.

- Encuestas.

- Videos.

- Papelotes.

- Transparencias.

\subsection{Evaluación}

Encuestas de opinión a alumnos y padres de familia, antes del inicio del programa y al final del mismo. 
